\title{
Mixed apocrine/endocrine ductal carcinoma in situ of the breast coexistent with lobular carcinoma in situ
}

\author{
J D Coyne, P A Dervan, L Barr, A D Baildam
}

\begin{abstract}
An unusual mixed form of ductal carcinoma in situ (DCIS) of the breast is described, which exhibits a biphenotypic morphology encompassing a range of differential diagnostic DCIS subtypes. In adddition, immunophenotypic and ultrastructural studies demonstrate neuroendocrine and apocrine differentiation, raising questions regarding appropriate classification and biological behaviour. In two cases, coexistence of this mixed form of DCIS with lobular carcinoma in situ (LCIS) in the same duct lobular units is an additional unusual feature that might, at least in some cases, indicate a closer relation between them.

(f Clin Pathol 2001;54:70-73)
\end{abstract}

Keywords: lobular carcinoma in situ; ductal carcinoma in situ; apocrine differentiation

Apocrine differentiation is common in invasive breast cancer ${ }^{1}$ and it is usually recognised by tumour cells containing granular pink cytoplasm. Less commonly, however, the cytoplasm is foamy or clear. In these circumstances, apocrine differentiation is defined by immunohistochemical and ultrastructural characteristics. $^{2}$ Although apocrine differentiation is not rare in ductal carcinoma in situ (DCIS), ${ }^{3}$ its manifestation with a predominantly clear cell morphology is unusual. Similarly, neuroendocrine differentiation has more recently been characterised by combined morphological, immunohistological, and ultrastructural characteristics, firmly establishing the existence of endocrine differentiation in DCIS. ${ }^{4}$ As a further illustratration of the heterogeneity possible in preinvasive breast neoplasia, we describe three unusual cases of DCIS showing dimorphic apocrine and neuroendocrine differentiation, two of which showed coexisting lobular carcinoma in situ (LCIS).

Surgery, Withington

Hospital

L Barr

A D Baildam

Department of

Pathology Mater

Hospital, Eccles

Street, Dublin 7,

Ireland

P A Dervan

Correspondence to: Dr Coyne

JOHN.COYNE@man.ac.uk

Accepted for publication 19 April 2000
Materials and methods

Tissues were fixed in $10 \%$ buffered formalin, routinely processed, and stained with haematoxylin and eosin. For immunohistochemistry, representative sections were selected and, using the avidin biotin technique, staining was performed for the following: E-cadherin (R\&D systems, Oxon, UK), S100 protein (Dako, Cambridgeshire, UK), chromogranin (Dako), synaptophysin (Dako), the oestrogen receptor (Novocastra, Newcastle, UK), the progesterone receptor (Novocastra), gross cystic disease fluid protein (GCDFP-15; Signet, Dedham, Massachusetts, USA), epithelial membrane antigen (EMA; Dako), $\alpha$ smooth muscle actin (SMA; Dako), neurone specific enolase (NSE; Dako), MIB1 (Immunotec, Marseilles, France). For electron microscopy, five selected pieces of tissue from case 1 were dewaxed and reprocessed after postfixation in osmium. Sections ( $1 \mu \mathrm{m}$ thick) were cut and stained with toluidine blue. Ultrathin sections were cut and stained with uranyl acetate and lead citrate, one of which was from a spindle cell area.

\section{Case histories and pathology}

CASE 1

A 45 year old woman had suspicious microcalcification on mammography. An excision biopsy measuring $4.0 \times 3.0 \times 1.0 \mathrm{~cm}$ was performed after needle localisation. Several additional margin shavings from the biopsy cavity were also removed. This was followed by a subcutaneous mastectomy six weeks later. A hysterectomy and bilateral salpingoophorectomy, two years later, showed adenomyosis. The patient is well and disease free five years after mastectomy.

Microscopic examination of most of the sections from the original biopsy, from the shavings, and from around the cavity of the mastectomy specimen showed similar features. The lesion was multifocal and showed coexistent DCIS and LCIS. The DCIS was heterogeneous with low, intermediate, and high grade areas. The tumour cells mostly displayed pink or clear cytoplasm. A cribriform pattern was focally present but a solid pattern predominated. Within the solid areas of low and intermediate grade, small gland-like spaces were sometimes seen and a spindle cell pattern was common (figs 1 and 2). Occasional organoid and trabecular foci were present (fig 3). Large vacuolated cells were prominent and were present at the periphery of ducts, but also

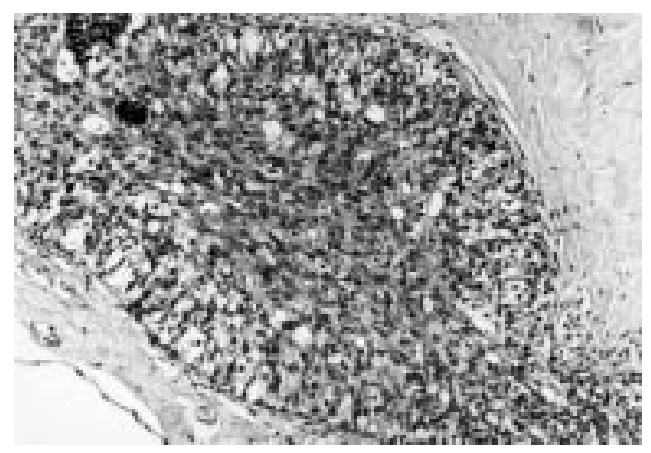

Figure 1 Spindle and clear cell ductal carcinoma in situ. 


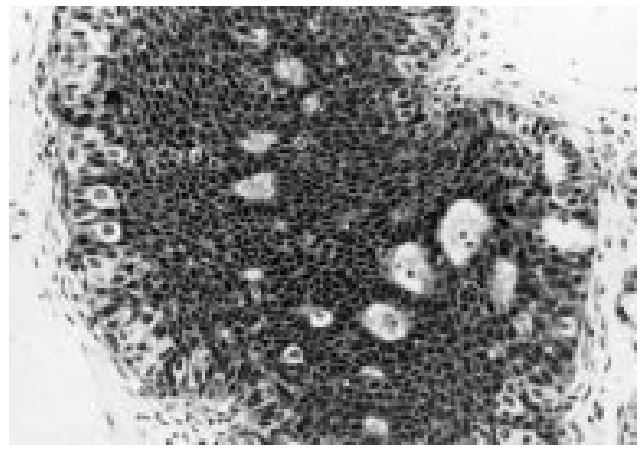

Figure 2 Ductal carcinoma in situ showing small glandular spaces and pseudorosette-like spaces.

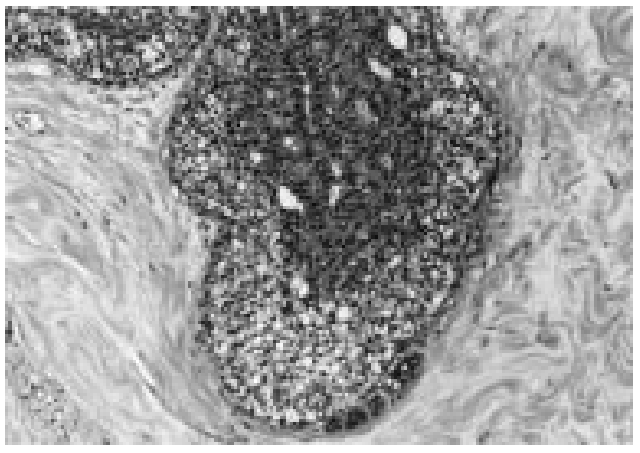

Figure 3 Clear cell ductal carcinoma in situ with a trabecular arrangement in places.

completely filled some of the duct lumina. Some ducts contained fibrovascular cores. Cancerisation of lobules was present and extensive pagetoid spread by vacuolated and non-vacuolated cells was also seen.

A few foci of obvious apocrine DCIS composed of cells with abundant eosinophilic granular cytoplasm were present (fig 4). Some areas contained histiocytoid cells with lightly eosinophilic, sparse cytoplasm. Some ducts showed a monomorphic appearance, whereas others displayed a variable appearance with a mixture of spindle cells, clear cells, and/or apocrine cells and occasional histiocytoid cells. The glands contained some diastase resistant, periodic acid Schiff (PAS)/alcian blue positive material. Occasional mitoses and small foci of necrosis and microcalcifications were apparent in high grade foci. Solid low and intermediate grade DCIS in places resembled epithelial hyperplasia, displaying small irregular spaces and cells with intracytoplasmic lumina. Extensive LCIS, comprising about one third of the lesion, was also present. Pagetoid spread and clover leaf structures were prominent. Intracytoplasmic lumina, some showing targetoid alcian blue/PAS positive mucin, were abundant and occasional larger vacuolated cells were also seen. In small acini containing LCIS, abrupt transition with histiocytoid cells occurred focally, as well as more frequent areas that showed merging of LCIS and large vacuolated cells. Within large ducts there was an abrupt transition between LCIS and DCIS of spindle, clear, and non-clear cell type. In most of these coexistent areas the LCIS was located centrally and, in places, the LCIS appeared to have been subjected to pagetoid spread by both vacuolated and non-vacuolated, larger DCIS-type

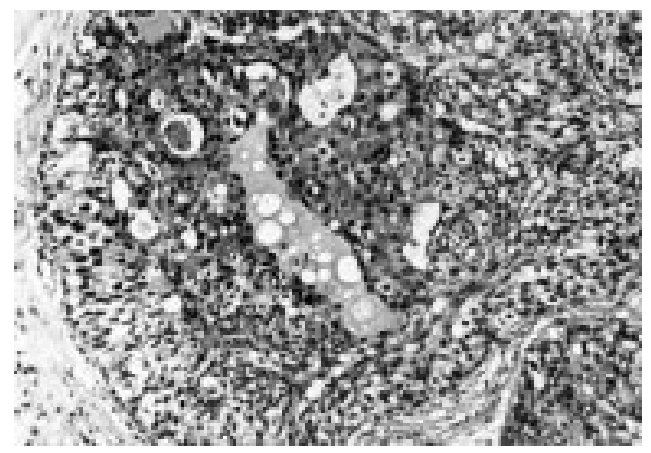

Figure 4 Clear cell ductal carcinoma in situ (DCIS) with adjacent area of conventional apocrine DCIS.

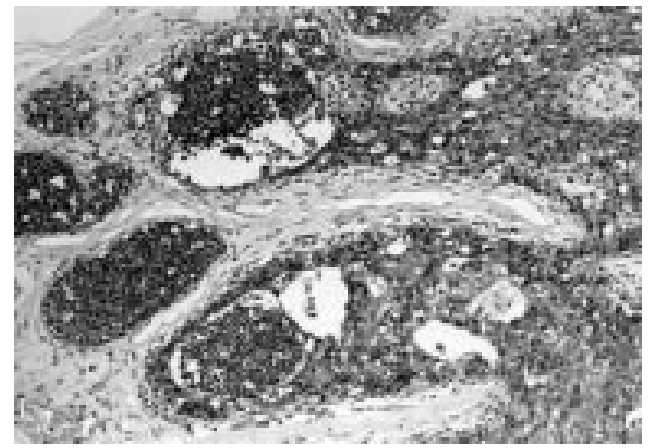

Figure 5 Coexistent ductal carcinoma in situ (DCIS) and lobular carcinoma in situ with pagetoid extension of DCIS.

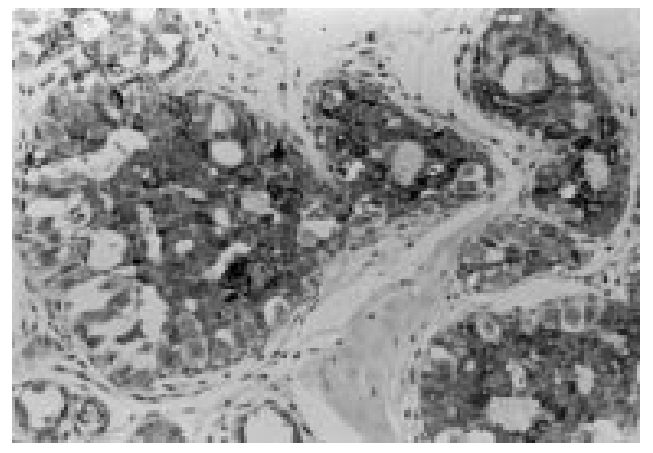

Figure 6 Patchy positivity for synaptophysin.

cells (fig 5). Transitional areas with features making it difficult to ascribe a lobular or ductal origin to them were also noted.

Immunohistochemistry gave the following results: strong positivity in both LCIS and DCIS components for cytokeratin and EMA. E-cadherin, NSE, and synaptophysin showed strong positivity in DCIS including clear, spindle, and apocrine components, but a negative reaction in the conventional LCIS component (figs 6 and 7). Many of the clear cells were positive with GCDFP-15 (fig 8), as were the apocrine cells and occasional LCIS cells; however, only some of the histiocytoid cells reacted. Strong positivity for oestrogen receptors was present in spindle cell DCIS, with patchy positivity in high grade and overtly apocrine areas. Progesterone receptors were only focally positive. The surrounding myoepithelial cells stained positively for $\mathrm{S} 100$ protein and SMA.

Ultrastructural examination was performed on dewaxed tissue taken from five blocks and 


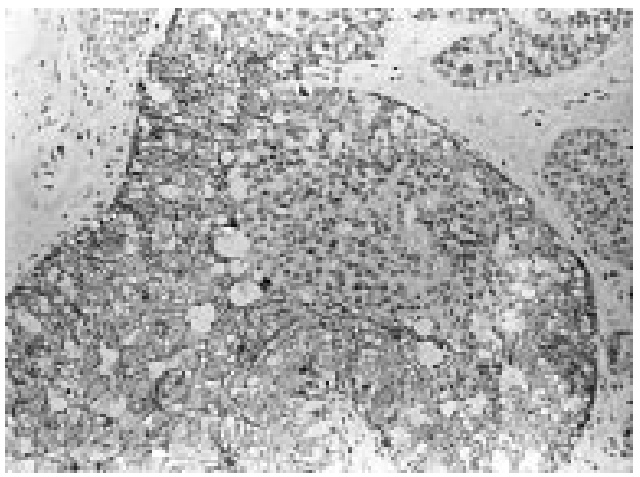

Figure 7 Coexistent ductal carcinoma in situ (DCIS) and lobular carcinoma in situ showing E-cadherin positivity in the DCIS component.

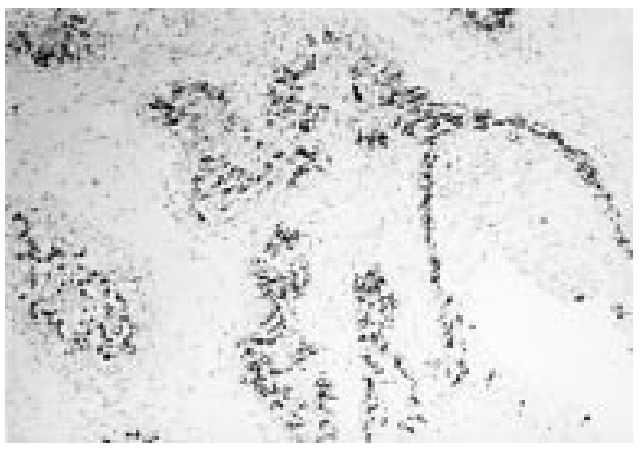

Figure 8 Positive reaction for gross cystic disease fluid protein (GCDFP-15) mainly in clear cells.

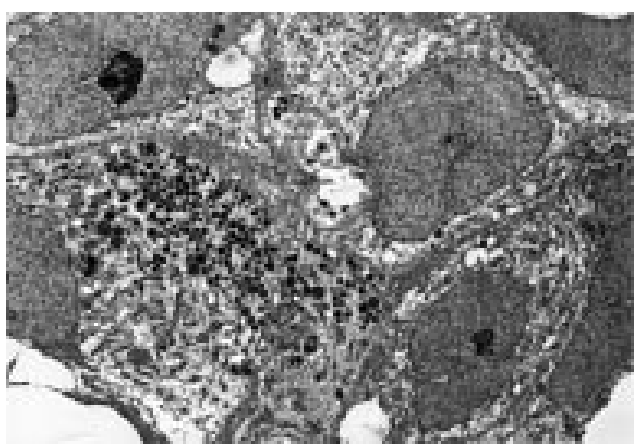

Figure 9 Ultrastructural presence of dense core type granules.

showed clear cells containing small empty vacuoles. One of the first samples, taken from a spindle cell area, also showed cells with large (300-700 nm) and small electron dense granules (100-140 nm). Attempts to carry out the uranaffin reaction were thwarted because further tissue samples were devoid of granules. Occasional cells contained both empty vacuoles and electron dense granules (fig 9). Cells with intracytoplasmic lumina were present, as were intercellular lumina, and cells containing small microvilli at one pole were also noted.

CASE 2

A 54 year old woman was found to have an irregular area of microcalcification in the upper left aspect of the left breast. A needle core biopsy showed DCIS and a needle localisation excision biopsy was performed. After this, she received radiotherapy and 12 months later she is well and disease free.
The biopsy specimen measured $4.0 \times 3.5 \times 2.5 \mathrm{~cm}$ and microscopic examination showed solid, micropapillary, and cribriform DCIS of intermediate grade measuring $15 \mathrm{~mm}$ in dimension. It displayed a mixed clear, spindle, and conventional morphology, with a mixed immunophenotype of apocrine and endocrine differentiation (chromogranin, synaptophysin, and GCDFP-15 positivity). A $2 \mathrm{~mm}$ focus of infiltrating ductal carcinoma, grade 1, was also present. Steroid receptor evaluation showed positivity for oestrogen receptors in almost all nuclei and weak positivity for progesterone receptors in a few nuclei.

CASE 3

A 61 year old woman was found to have suspicious microcalcification in the right breast on mammography. Fine needle aspiration cytology showed malignant cells and a needle localised wide local excision was performed. Because DCIS and invasive malignancy extended to the excision margins, a right mastectomy was performed three weeks later. The patient is well 13 months after mastectomy.

The biopsy consisted of a piece of tissue measuring $3.5 \times 3.0 \times 2.0 \mathrm{~cm}$. Microscopic examination showed admixed intermediate grade DCIS, foci of coexistent LCIS, and infiltrating ductal carcinoma, grade 2 , measuring $1.5 \mathrm{~cm}$. The DCIS component showed a variably mixed pattern of clear and spindle cells with occasional pseudorosettes. The invasive component also showed a variable appearance, with both solid nests and focal cribriform areas with clear cell change. Oestrogen receptors were strongly positive in almost all nuclei and synaptophysin, chromagranin, and GCDFP-15 were strongly positive within both the DCIS and the invasive components.

\section{Discussion}

The reported incidence of apocrine differentiation in breast cancer has varied greatly depending on defining characteristics. ${ }^{5}$ In the past, apocrine differentiation was recognised by the characteristic granular, eosinophilic cytoplasm. The recognition of foamy and sometimes clear cell morphologies, with immunohistochemical reactivity for GCDFP15 , and the ultrastructural presence of empty vesicles has increased the awareness, identification, and incidence of apocrine differentiation. ${ }^{267}$ In fact, Eusebi et al consider apocrine differentiation in DCIS to be one of the most under recognised changes in breast pathology, quoting nine of 20 cases of poorly differentiated DCIS as showing GCDFP-15 reactivity. However, when composed predominantly of clear cells, the apocrine nature of DCIS may be even less readily apparent. Clear cells can also be a feature of neuroendocrine breast carcinomas, ${ }^{5}$ but they are not a prominent feature in neuroendocrine DCIS. ${ }^{4}$ Despite recent clarification, ${ }^{5}$ the issue of neuroendocrine breast carcinoma remains controversial. ${ }^{8}$ However, the existence of neuroendocrine DCIS as a distinct category, although rare, appears to be well established. ${ }^{4}$ It was first systematically 
investigated by Cross et al using typical morphological features, argyrophilia, immunocytochemistry, and ultrastructural findings. ${ }^{9}$ Two further cases were described by Ashworth and Haqqani, ${ }^{10}$ and Maluf et al added a further two cases, one containing mucinous areas. ${ }^{11}$ Maluf and Koerner described a further 20 cases of neuroendocrine DCIS, which they designated as solid papillary carcinoma, and surprisingly state that special studies are not required for evidence of endocrine differentiation. ${ }^{12}$ Recently Tsang and Chan, in the largest case series of endocrine DCIS to date, further refined this entity with associated distinctive biological implications and defined the diagnostic criteria for diagnosis. ${ }^{4}$

We believe our cases fulfil the criteria for both neuroendocrine and apocrine differentiation in DCIS, with case 3 also showing these features in the invasive component. The light microscopic presence of clear cells and focal typical apocrine morphology, together with GCDFP-15 immunoreactivity and the ultrastructural presence of empty vesicles, indicate apocrine differentiation in DCIS. The variable combination of spindle cell morphology, small glandular and rosette-like spaces, polypoid protrusions, prominent pagetoid spread, the presence of fibrovascular cores, clear cells, and immunohistochemical positivity for synaptophysin and NSE strongly support neuroendocrine differentiation in DCIS and invasive carcinoma. In addition, the presence of small and large electron dense granules support this contention, although we acknowledge that such granules as those in case 1 do not always indicate neuroendocrine differentiation. ${ }^{13}$

The differential diagnosis of predominantly clear and spindle cell DCIS includes both apocrine and neuroendocrine DCIS, myoepithelial DCIS, and presumably intraductal variants of glycogen and lipid rich carcinomas, as well as fixation artefact. ${ }^{2}$ As distinct entities, apocrine and neuroendocrine DCIS are not uncommon, but their occurrence as a mixed or combined subtype of DCIS with an unusual dimorphic appearance appears to be a rare event. We believe the bidirectional differentiation to be truly mixed because of overlapping features in both immunohistochemistry and ultrastructure. Although there is difficulty in definitively ascribing individual cells to one category or another, there may be validity in proposing that the clear and spindle cells demonstrate more apocrine and neuroendocrine features, respectively. To some extent, the morphology resembled the intraductal malignant myoepithelioma described by Tamai, the cells of which however are positive for S100 protein and smooth muscle actin. ${ }^{14}$

Coexistent LCIS and DCIS is itself a rare phenomenon $^{15}$ and is remarkably present in two of these cases. Although of uncertain histogenesis, the coexistence of two patterns of non-invasive carcinoma is postulated to arise as a result of a "collision carcinoma" or pagetoid growth. ${ }^{15}$ If carcinoma arises from the terminal ductule/lobular unit one would expect these combinations to occur. The very focal presence of histiocytoid carcinoma, an apocrine variant of LCIS, ${ }^{16}$ associated with the LCIS in case 1 might suggest a closer relation between coexistent DCIS and LCIS, despite neuroendocrine differentiation being restricted to the DCIS component.

These cases extend the spectrum of neuroendocrine DCIS, demonstrate the heterogeneity of differentiation possible in DCIS, and may indicate a closer relation, at least in some cases, between DCIS and LCIS as different patterns of preinvasive breast cancers. These results support the concept of including and separately categorising rare types of DCIS in classification systems to ascribe biological importance to unusual variants including mixed patterns.

We thank Dr A Curry and Miss P Rowlands for electron and photomicrographs and Miss J Metcalfe and Mrs V Mistry for secretarial assistance.

1 Rosen PP. Rosen's breast pathology. Philadelphia: LippincottRaven, 1996.

2 Dina R, Eusebi V. Clear cell tumours of the breast. Semin Diagn Pathol 1997;14:175-82.

3 Eusebi V, Daminani S, Losi L, et al. Apocrine differentiation in breast epithelium. Adv Anat Pathol 1997;4:139-55.

4 Tsang YWW, Chan JKC. Endocrine ductal carcinoma in-situ (E-DCIS) of the breast; a form of low-grade DCIS with distinctive clinicopathologic and biologic characteriswith distinctive clinicopathologic and biold
tics. Am F Surg Pathol 1996;20:921-43.

5 Papotti M, Macri L, Finzi G, et al. Neuroendocrine differentiation in carcinomas of the breast: a study of 51 cases. Semin Diagn Pathol 1989;6:174-188.

6 Eusebi V, Millis RR, Cattani MG, et al. Apocrine carcinoma of the breast; a morphologic and immunocytochemical study. Am F Pathol 1986;123:532-41.

7 Mazoujian G, Pinkus GS, Davis S, et al. Immunohistochemistry of gross cystic disease fluid protein (GCDFP-15) of the breast; a marker of apocrine epithelium and breast carcinomas with apocrine features. Am f Surg Pathol 1983;110:105-12.

8 Ellis IO, Elston CW. Tumours of the breast. In: Fletcher CDM, ed. Diagnostic histopathology of tumours, Vol. I. Edinburgh: Churchill Livingstone 1995:635-88.

9 Cross AS, Azzopardi JG, Krausz T, et al. A morphological and immunocytochemical study of a distinctive variant of ductal carcinoma in-situ of the breast. Histopathology 1985; 9:21-37.

10 Ashworth MT, Haqqani MT. Endocrine variant of ductal carcinoma in-situ of breast: ultrastructural and light microscopical study. F Clin Pathol 1986;39:1355-9.

11 Maluf HM, Zukerberg LR, Dickersin GR, et al. Spindle-cell argyrophilic mucin-producing carcinoma of the breast. $\mathrm{Am}$ argyrophilic mucin-producing

12 Maluf HM, Koerner FC. Solid papillary carcinoma of the breast; a form of intraductal carcinoma with endocrine differentiation frequently associated with m
noma. Am f Surg Pathol 1995;19:1237-44.

13 Battersby S, Dely CJ, Hopkinson HE, et al. The nature of breast dense core granules: chromogranin reactivity. Histopathology 1992;20:107-14.

14 Tamai $M$. Intraductal growth of malignant mammary myoepithelioma. Am $\mathcal{F}$ Surg Pathol 1992;16:1116-25.

15 Rosen PP. Coexistent lobular carcinoma in-situ and intraductal carcinoma in a single lobular-duct unit. $A m \mathcal{F}$ Surg Pathol 1980;4:241-6.

16 Walford N, Ten Velden J. Histiocytoid breast carcinoma: an apocrine variant of lobular carcinoma. Histopathology 1989; 14:515-22. 